\title{
Axiomatic Foundations of Multiplier Preferences
}

\section{Citation}

Strzalecki, Tomasz. 2011. "Axiomatic Foundations of Multiplier Preferences." Econometrica 79 (1): 47-73.

\section{Published Version}

doi:10.3982/ECTA8155

\section{Permanent link}

http://nrs.harvard.edu/urn-3:HUL.InstRepos:14397610

\section{Terms of Use}

This article was downloaded from Harvard University's DASH repository, and is made available under the terms and conditions applicable to Other Posted Material, as set forth at http:// nrs.harvard.edu/urn-3:HUL.InstRepos:dash.current.terms-of-use\#LAA

\section{Share Your Story}

The Harvard community has made this article openly available.

Please share how this access benefits you. Submit a story.

Accessibility 


\section{ECONOMETRICA}

JOURNAL OF THE ECONOMETRIC SOCIETY

An International Society for the Advancement of Economic Theory in its Relation to Statistics and Mathematics

http://www.econometricsociety.org/

Econometrica, Vol. 79, No. 1 (January, 2011), 47-73

\section{AXIOMATIC FOUNDATIONS OF MULTIPLIER PREFERENCES}

TOMASZ STRZALECKI

Harvard University, Cambridge, MA 02138, U.S.A.

The copyright to this Article is held by the Econometric Society. It may be downloaded, printed and reproduced only for educational or research purposes, including use in course packs. No downloading or copying may be done for any commercial purpose without the explicit permission of the Econometric Society. For such commercial purposes contact the Office of the Econometric Society (contact information may be found at the website http://www.econometricsociety.org or in the back cover of Econometrica). This statement must be included on all copies of this Article that are made available electronically or in any other format. 


\section{AXIOMATIC FOUNDATIONS OF MULTIPLIER PREFERENCES}

\section{BY TOMASZ STRZALECKI ${ }^{1}$}

This paper axiomatizes the robust control criterion of multiplier preferences introduced by Hansen and Sargent (2001). The axiomatization relates multiplier preferences to other classes of preferences studied in decision theory, in particular, the variational preferences recently introduced by Maccheroni, Marinacci, and Rustichini (2006a). This paper also establishes a link between the parameters of the multiplier criterion and the observable behavior of the agent. This link enables measurement of the parameters on the basis of observable choice data and provides a useful tool for applications.

KEYWORDS: Ambiguity aversion, model uncertainty, robustness.

\section{INTRODUCTION}

THE EXPECTED UTILITY CRITERION ranks payoff profiles $f$ according to

$$
V(f)=\int u(f) d q,
$$

where $u$ is a utility function and $q$ is a subjective probability distribution on the states of the world. A decision maker with such preferences behaves as if he is certain that the state is distributed according to the probabilistic model $q$.

To model situations where the decision maker does not have enough information to formulate a single probabilistic model and have full confidence in it, for example, when it is hard to statistically distinguish between similar probabilistic models, Hansen and Sargent (2001) formulated the criterion

$$
V(f)=\min _{p} \int u(f) d p+\theta R(p \| q)
$$

where $\theta \in(0, \infty]$ is a parameter and the function $R(p \| q)$ is the relative entropy of $p$ with respect to $q$. Relative entropy, otherwise known as Kullback-Leibler divergence, is a measure of "distance" between two probability distributions.

\footnotetext{
${ }^{1}$ I am indebted to my advisor, Eddie Dekel, for his continuous guidance, support, and encouragement. I am grateful to Peter Klibanoff and Marciano Siniscalchi for many discussions which resulted in significant improvements of the paper. I would also like to thank Jeff Ely, Todd Sarver, and seminar audiences at Berkeley, Bocconi, CEMFI, Chicago, Collegio Carlo Alberto, Columbia, Duke (Fuqua), Harvard, Hebrew University, LSE, NYU (Econ and Stern), Northwestern, Penn, Princeton, Stanford (Econ and GSB), UCL, University of Iowa, University of Minnesota, Warwick, Washington University (Econ and Olin), Yale, and the Hansen-Sargent conference for graduate students. This project started after a very stimulating conversation with Tom Sargent and was further shaped by conversations with Lars Hansen. I am very grateful to the co-editor and three anonymous referees for their insightful and helpful comments. All errors are my own.
} 
An interpretation of equation (2) is that the decision maker has some best guess $q$ of the true probability distribution, but does not fully trust it. Instead, he considers many other probabilities $p$ to be plausible, with plausibility diminishing proportionally to their "distance" from $q$. The role of the proportionality parameter $\theta$ is to measure the degree of trust of the decision maker in the reference probability $q$ or, in other words, the concern for model misspecification. Higher values of $\theta$ correspond to more trust; in the limit, with the convention that $0 \cdot \infty=0$ when $\theta=\infty$, the decision maker fully trusts his reference probability $q$ and uses the expected utility criterion (1).

Multiplier preferences (2) also belong to the more general class of variational preferences studied by Maccheroni, Marinacci, and Rustichini (2006a), which have the representation

$$
V(f)=\min _{p} \int u(f) d p+c(p) .
$$

The interpretation of (3) is like that of (2), and multiplier preferences are a special case of variational preferences with $c(p)=\theta R(p \| q)$. In general, the conditions that the function $c(p)$ in (3) has to satisfy are very weak, which makes variational preferences a very broad class. In addition to expected utility preferences and multiplier preferences, this class also nests the maxmin expected utility preferences of Gilboa and Schmeidler (1989), as well as the mean-variance preferences of Markowitz (1952) and Tobin (1958).

An important contribution of Maccheroni, Marinacci, and Rustichini (2006a) was to provide an axiomatic characterization of variational preferences. Because variational preferences are a very broad class of preferences, it is desirable to establish an observable distinction between multiplier preferences and other subclasses of variational preferences. This is, for example, the case with the maxmin expected utility preferences of Gilboa and Schmeidler (1989): a strengthening of the Maccheroni, Marinacci, and Rustichini (2006a) axioms restricts the general function $c(p)$ to be in the Gilboa and Schmeidler (1989) class.

The main finding of this paper is that the sure-thing principle of Savage (imposed on the Anscombe-Aumann domain) characterizes the class of multiplier preferences within the class of variational preferences. This is possible because, as the main theorem shows, the class of multiplier preferences is precisely the intersection of the class of variational preferences and the class of second-order expected utility (SOEU) preferences with representation

$$
V(f)=\int \phi(u(f)) d q
$$




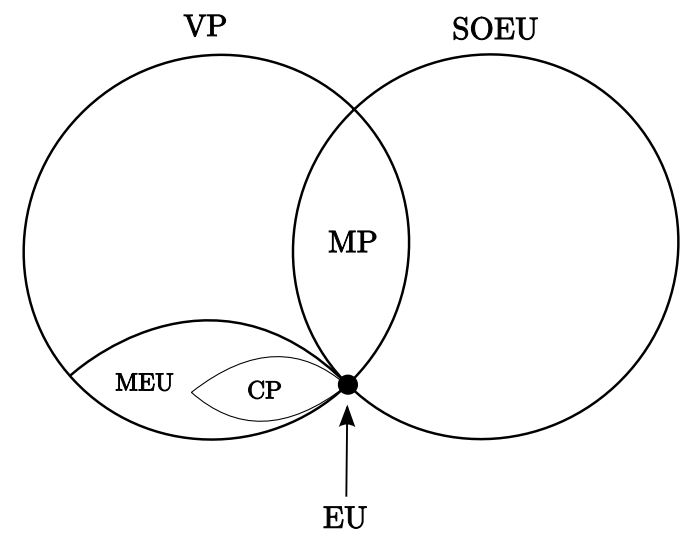

FIGURE 1.-Relations between classes of preferences: VP, variational preferences; MP, multiplier preferences; SOEU, second-order expected utility preferences; EU, expected utility preferences; MEU, maxmin expected utility preferences; $\mathrm{CP}$, constraint preferences.

for some real-valued function $\phi .^{2}$ Figure 1 depicts the relationships between these classes. ${ }^{3}$ The sure thing principle axiom used in the characterization is standard in the literature; in particular, it is not in any way related to the very specific functional form of relative entropy-it is the interaction between the axioms that delivers the representation.

The proposed axiomatic characterization is important for three reasons. First, it provides a set of testable predictions of the model that allow for its empirical verification. This will help evaluate whether multiplier preferences, which have already proved useful in modeling behavior at the macro level, ${ }^{4}$ are an accurate model of individual behavior. Second, the axiomatization establishes a link between the parameters of the multiplier criterion and the observable behavior of the agent. This link enables measurement of the parameters on the basis of observable choice data alone, without relying on unverifiable assumptions. Finally, the axiomatization is helpful in understanding the relation between the multiplier preferences and the axiomatic models of ambiguity aversion motivated by the Ellsberg (1961) paradox, where people exhibit a preference for choices involving objective rather than subjective probabilities.

${ }^{2}$ For axiomatic characterizations of such preferences, see Neilson (1993, 2010), Nau (2001, 2006), Ergin and Gul (2009), and Grant, Polak, and Strzalecki (2009).

${ }^{3}$ Hansen and Sargent also introduced a closely related class of constraint preferences, represented by $V(f)=\min _{\{p \mid R(p \| q) \leq \eta\}} \int_{S}(u \circ f) d p$, which are a special case of Gilboa and Schmeidler's (1989) maxmin expected utility preferences; see Figure 1. Due to their greater analytical tractability, multiplier-rather than constraint-preferences are used in applications.

${ }^{4}$ See Barillas, Hansen, and Sargent (2009), Benigno and Nisticò (2009), Hansen, Sargent, and Tallarini (1999), Hansen, Sargent, and Wang (2002), Karantounias, Hansen, and Sargent (2009), Kleshchelski and Vincent (2009), Li and Tornell (2008), Maenhout (2004), and Woodford (2006). 
The paper is organized as follows. Section 2 introduces some notation and basic concepts, as well as the definition of multiplier preferences. Section 3 presents an axiomatic characterization of multiplier preferences within the class of variational preferences in the classic setting of Anscombe and Aumann. Section 4 studies another choice domain, introduced by Ergin and Gul (2009), and presents a fully subjective axiomatization of multiplier preferences. Section 5 concludes.

\section{PRELIMINARIES}

\subsection{Setup}

Decision problems considered in this paper involve a set $S$ of states of the world, which represents the possible contingencies that may occur. One of the states, $s \in S$, will be realized, but the decision maker has to choose the course of action before learning $s$. Let $\Sigma$ denote a sigma-algebra of events in $S .{ }^{5}$ The set of all finitely additive probability measures on $(S, \Sigma)$ is denoted $\Delta(S)$ and endowed with the weak* topology, where a net $\left\{p_{d}\right\}_{d \in D}$ converges to $p$ if $p_{d}(A) \rightarrow p(A)$ for all $A \in \Sigma$; the set of all countably additive probability measures is denoted $\Delta^{\sigma}(S)$; its subset that consists of all measures absolutely continuous with respect to $q \in \Delta^{\sigma}(S)$, is denoted $\Delta^{\sigma}(q)$.

The set $Z$ denotes the possible consequences and $\Delta(Z)$ denotes simple probability distributions on $Z$. An element of $\Delta(Z)$ is called a lottery. A lottery paying off $z \in Z$ with probability 1 is denoted $\delta_{z}$. For any two lotteries $\pi, \pi^{\prime} \in \Delta(Z)$ and a number $\alpha \in(0,1)$, the lottery $\alpha \pi+(1-\alpha) \pi^{\prime}$ assigns probability $\alpha \pi(z)+(1-\alpha) \pi^{\prime}(z)$ to each prize $z \in Z$.

The alternatives that the decision maker faces, called acts, are mappings from $S$ to $\Delta(Z){ }^{6}$ Formally, an act is a finite-valued, $\Sigma$-measurable function $f: S \rightarrow \Delta(Z)$; the set of all such acts is denoted $\mathcal{F}(\Delta(Z))$. If $f, g \in \mathcal{F}(\Delta(Z))$ and $E \in \Sigma$, then $f_{E} g$ denotes an act with $f_{E} g(s)=f(s)$ if $s \in E$ and $f_{E} g(s)=$ $g(s)$ if $s \notin E$. If $f, g \in \mathcal{F}(\Delta(Z))$ and $\alpha \in[0,1]$, then $\alpha f+(1-\alpha) g$ denotes an act that assigns the lottery $\alpha f(s)+(1-\alpha) g(s)$ in each state $s \in S$.

The choices of the decision maker are represented by a preference relation $\succsim$, where $f \succsim g$ means that the act $f$ is weakly preferred to the act $g$. A functional $V: \mathcal{F}(\Delta(Z)) \rightarrow \mathbb{R}$ represents $\succsim$ if for all $f, g \in \mathcal{F}(\Delta(Z))$,

$$
f \succsim g \text { if and only if } V(f) \geq V(g) .
$$

An important class of preferences are the expected utility (EU) preferences, where the decision maker has a probability distribution $q \in \Delta(S)$ and a utility function that evaluates each consequence $u: Z \rightarrow \mathbb{R}$. A preference relation $\succsim$

\footnotetext{
${ }^{5}$ The set $S$ may be infinite or finite. When $S$ is finite, it is assumed that $\Sigma=2^{S}$.

${ }^{6}$ This setting was introduced by Fishburn (1970). Settings of this type are usually named after Anscombe and Aumann (1963), who were the first to work with them.
} 
has an EU representation $(u, q)$ if a functional $V: \mathcal{F}(\Delta(Z)) \rightarrow \mathbb{R}$ represents $\succsim$, where

$$
V(f)=\int_{S}\left(\sum_{z \in Z} u(z) f(s)(z)\right) d q(s) .
$$

In each state of the world $s$, the decision maker computes the expected utility of the lottery $f(s)$ and then averages those values across states. By slightly abusing notation, define the affine function $u: \Delta(Z) \rightarrow \mathbb{R}$ by $u(\pi)=\sum_{z \in Z} u(z) \pi(z)$. Using this definition, the expected utility criterion can be written as

$$
V(f)=\int_{S} u(f(s)) d q(s) .
$$

Risk aversion is the phenomenon where sure payoffs are preferred to payoffs that are stochastic but have the same expected monetary value. If $Z=\mathbb{R}$, that is, lotteries have monetary payoffs, then risk averse EU preferences have concave utility functions $u$. Likewise, one preference relation is more risk averse than another if it has a "more concave" utility function. Formally, an EU preference represented by $\left(u_{1}, q_{1}\right)$ is more risk averse than one represented by $\left(u_{2}, q_{2}\right)$ if and only if $q_{1}=q_{2}$ and $u_{1}=\phi \circ u_{2}$, where $\phi: \mathbb{R} \rightarrow \mathbb{R}$ is a strictly increasing concave transformation. A special role will be played by the class of transformations $\phi_{\theta}$, indexed by $\theta \in(0, \infty]$ :

$$
\phi_{\theta}(u)= \begin{cases}-\exp \left(-\frac{u}{\theta}\right) & \text { for } \theta<\infty \\ u & \text { for } \theta=\infty\end{cases}
$$

Lower values of $\theta$ correspond to more concave transformations, that is, more risk aversion.

\subsection{Sources of Uncertainty and the Ellsberg Paradox}

Observe that every act $f: S \rightarrow \Delta(Z)$ involves two sources of uncertainty: first, the payoff of $f$ is contingent on the state of the world, for which there is no objective probability given; second, given the state, $f(s)$ is an objective lottery.

The existence of two sources of uncertainty enables a distinction between purely objective lotteries, that is, acts which pay the same lottery $\pi \in \Delta(Z)$ irrespective of the state of the world, and purely subjective acts, that is, acts that in each state of the world pay off a degenerate lottery $\delta_{z}$ for some $z \in Z$, which possibly depends on $s$. With a slight abuse of notation, let $\Delta(Z)$ denote the set of purely objective lotteries. Note that given $q \in \Delta(S)$, each purely subjective act $f$ induces a purely objective lottery $\pi_{f} \in \Delta(Z)$ defined by $\pi_{f}(z)=q\left(f^{-1}(z)\right)$ for all $z \in Z$. 
An EU decision maker has the same attitude toward objective lotteries and toward subjective acts. From the representation (5), it follows that for any two purely objective lotteries $\pi^{\prime} \succsim \pi$ if and only if

$$
\sum_{z \in Z} u(z) \pi^{\prime}(z) \geq \sum_{z \in Z} u(z) \pi(z),
$$

and for any two purely subjective acts $f^{\prime} \succsim f$ if and only if

$$
\sum_{z \in Z} u(z) \pi_{f^{\prime}}(z) \geq \sum_{z \in Z} u(z) \pi_{f}(z) .
$$

In particular, any purely subjective act $f$ is indifferent to the objective lottery $\pi_{f}$ that it induces.

However, more general preferences need not have such a uniform decision attitude and they may be source sensitive, that is, exhibit more aversion to one source than the other. This is illustrated by the Ellsberg (1961) paradox, which demonstrates that most people prefer choices involving risk (i.e., situations in which the probability is well specified) to choices involving ambiguity (where the probability is not specified). ${ }^{7}$

EXAMPLE 1-Ellsberg Paradox: Consider two urns containing colored balls. The decision maker can bet on the color of the ball drawn from each urn. Urn I contains 100 red and black balls in unknown proportion, while urn II contains 50 red and 50 black balls.

In this situation, most people are indifferent between betting on red from urn I and on black from urn I; this reveals that, in the absence of evidence against symmetry, they view those two contingencies as interchangeable. Moreover, most people are indifferent between betting on red from urn II and on black from urn II; this preference is justified by their knowledge of the composition of urn II. However, most people strictly prefer betting on red from urn II to betting on red from urn I, thereby displaying ambiguity aversion.

Ambiguity aversion cannot be reconciled with the EU model. To see that, let the state space $S=\{R, B\}$ represent the possible draws from urn I.

Betting $\$ 100$ on red from urn I corresponds to an act $f_{R}=\left(\delta_{100}, \delta_{0}\right)$, while betting $\$ 100$ on black from urn I corresponds to an act $f_{B}=\left(\delta_{0}, \delta_{100}\right)$. On the other hand, betting $\$ 100$ on red from urn II corresponds to a lottery $\pi_{R}=$ $\frac{1}{2} \delta_{100}+\frac{1}{2} \delta_{0}$, while betting $\$ 100$ on black from urn II corresponds to a lottery $\pi_{B}=\frac{1}{2} \delta_{0}+\frac{1}{2} \delta_{100}$. These correspondences reflect the fact that betting on urn I involves subjective uncertainty, while betting on urn II involves objective risks. Note in particular that $\pi_{R}=\pi_{B}$.

\footnotetext{
${ }^{7}$ Other experimental evidence on source sensitivity includes Abdellaoui, Baillon, Placido, and Wakker (2010), Chipman (1960), Curley and Yates (1989), Einhorn and Hogarth (1985), Fox and Tversky (1995), and Heath and Tversky (1991).
} 
Suppose that the subjective probability of drawing red from urn I is $q$ and drawing black from urn I is $1-q$. Observe that $V\left(\pi_{R}\right)=V\left(\pi_{B}\right)=\frac{1}{2} u(100)+$ $\frac{1}{2} u(0)$, whereas $V\left(f_{R}\right)=q u(100)+(1-q) u(0)$ and $V\left(f_{B}\right)=(1-q) u(100)+$ $q u(0)$. Because of the indifference $V\left(f_{R}\right)=V\left(f_{B}\right)$, it follows that $q=\frac{1}{2}$; hence, $V\left(f_{R}\right)=V\left(f_{B}\right)=\frac{1}{2} u(100)+\frac{1}{2} u(0)$. It follows that $\pi_{B} \sim \pi_{R} \sim f_{R} \sim f_{B}$, contradicting the typical Ellsberg choices.

As the above example shows, the Ellsberg pattern of choices cannot be explained by a model with a unique probability measure and with uniform aversion to both sources. In the literature there have been two main approaches to this problem. The first one replaces the probability measure $q$ with some other measure of belief that captures the decision maker's lack of information about the source; see, for example, the Choquet model of Schmeidler (1989), maxmin model of Gilboa and Schmeidler (1989), variational preferences of Maccheroni, Marinacci, and Rustichini (2006a), or smooth preferences of Klibanoff, Marinacci, and Mukherji (2005). The other approach is to keep $q$ but to introduce another parameter that captures the higher aversion toward variability coming from one source than another, as in the SOEU model (Ergin and Gul (2009), Grant, Polak, and Strzalecki (2009), Gul and Pesendorfer (2010), Nau (2001, 2006), Neilson (1993, 2010)). As this paper shows (see Section 3.3), the multiplier preferences have both representations and for this reason they belong to both families of models.

\subsection{Multiplier Preferences}

Hansen and Sargent (2001) and Hansen, Sargent, Turmuhambetova, and Williams (2006) introduced the class of multiplier preferences where the decision maker does not know the true probabilistic model $p$, but has a "best guess" or approximating model $q$, also called the reference probability. The decision maker thinks that the true probability $p$ is somewhere near the reference probability $q$. The notion of distance used by Hansen and Sargent is relative entropy.

DEFINITION 1: Let a reference measure $q \in \Delta^{\sigma}(S)$ be fixed. The relative entropy $R(\cdot \| q)$ is a mapping from $\Delta(S)$ into $[0, \infty]$ defined by

$$
R(p \| q)= \begin{cases}\int_{S}\left(\log \frac{d p}{d q}\right) d p, & \text { if } p \in \Delta^{\sigma}(q), \\ \infty, & \text { otherwise. }\end{cases}
$$

A decision maker who is concerned with model misspecification computes his expected utility according to all probabilities $p$, but he does not treat them equally. Probabilities closer to his best guess have more weight in his decision. 
DEFINITION 2: A relation $\succsim$ has a multiplier representation if it is represented by

$$
V(f)=\min _{p \in \Delta(S)} \int_{S} u(f(s)) d p(s)+\theta R(p \| q),
$$

where $u: \Delta(Z) \rightarrow \mathbb{R}$ is a nonconstant affine function, $\theta \in(0, \infty]$, and $q \in$ $\Delta^{\sigma}(S)$. In this case, $\succsim$ is called a multiplier preference.

The multiplier representation of $\succsim$ may suggest the following interpretation. First, the decision maker chooses an act without knowing the true distribution $p$. Second, "Nature" chooses the probability $p$ so as to minimize the decision maker's expected utility. Nature is not free to choose, but it incurs a "cost" for using each $p$. Probabilities $p$ that are farther from the reference measure $q$ have a larger potential for lowering the decision maker's expected utility, but Nature has to incur a larger cost to select them.

This interpretation suggests that a decision maker with such preferences is concerned with model misspecification and makes decisions that are robust to such misspecification. He is pessimistic about the outcome of his decision which leads him to exercise caution in choosing the course of action. Such cautious behavior is reminiscent of the Ellsberg paradox, as in Example 1 above. In fact, as Example 2 in Section 3.4.2 shows, multiplier preferences can be used to model such behavior.

\section{AXIOMATIZATION WITH OBJECTIVE RISK}

\subsection{Variational Preferences}

To capture ambiguity aversion, Maccheroni, Marinacci, and Rustichini (2006a) (henceforth MMR) introduced the class of variational preferences, with representation

$$
V(f)=\min _{p \in \Delta S} \int_{S} u(f(s)) d p+c(p),
$$

where $c: \Delta S \rightarrow[0, \infty]$ is a cost function.

Multiplier preferences are a special case of variational preferences where $c(p)=\theta R(p \| q)$. The variational criterion (7) can be given the same interpretation as the multiplier criterion (2): Nature wants to reduce the decision maker's expected utility by choosing a probability distribution $p$, but she is not entirely free to choose. Using different $p$ 's leads to different values of the decision maker's expected utility $\int_{S} u(f(s)) d p$, but comes at a cost $c(p)$.

To characterize variational preferences behaviorally, MMR used the following axioms.

AXIOM A1-Weak Order: The relation $\succsim$ is transitive and complete. 
AXIOM A2-Weak Certainty Independence: For all $f, g \in \mathcal{F}(\Delta(Z)), \pi, \pi^{\prime} \in$ $\Delta(Z)$, and $\alpha \in(0,1)$,

$$
\begin{aligned}
& \alpha f+(1-\alpha) \pi \succsim \alpha g+(1-\alpha) \pi \\
& \quad \Rightarrow \quad \alpha f+(1-\alpha) \pi^{\prime} \succsim \alpha g+(1-\alpha) \pi^{\prime} .
\end{aligned}
$$

AxIOM A3-Continuity: For any $f, g, h \in \mathcal{F}(\Delta(Z))$, the sets $\{\alpha \in[0,1] \mid \alpha f+$ $(1-\alpha) g \succsim h\}$ and $\{\alpha \in[0,1] \mid h \succsim \alpha f+(1-\alpha) g\}$ are closed.

AxIOM A4-Monotonicity: If $f, g \in \mathcal{F}(\Delta(Z))$ and $f(s) \succsim g(s)$ for all $s \in S$, then $f \succsim g$.

AXIOM A5-Uncertainty Aversion: If $f, g \in \mathcal{F}(\Delta(Z))$ and $\alpha \in(0,1)$, then

$$
f \sim g \quad \Rightarrow \quad \alpha f+(1-\alpha) g \succsim f .
$$

AXIOM A6-Nondegeneracy: $f \succ g$ for some $f, g \in \mathcal{F}(\Delta(Z))$.

AxIOM A8 - Weak Monotone Continuity: If $f, g \in \mathcal{F}(\Delta(Z)), \pi \in \Delta(Z)$, $\left\{E_{n}\right\}_{n \geq 1} \in \Sigma$ with $E_{1} \supseteq E_{2} \supseteq \cdots$, and $\bigcap_{n \geq 1} E_{n}=\emptyset$, then $f \succ g$ implies that there exists $n_{0} \geq 1$ such that $\pi E_{n_{0}} f \succ g$.

MMR showed that the preference $\succsim$ satisfies Axioms A1-A6 if and only if $\succsim$ is represented by (7) with an affine and nonconstant $u: \Delta(Z) \rightarrow \mathbb{R}$ and $c: \Delta S \rightarrow[0, \infty]$ that is convex, lower semicontinuous, and grounded (achieves value zero). Moreover, Axiom A8 guarantees that function $c$ is concentrated only on countably additive measures (observe, that Axiom A8 holds trivially if $S$ is finite).

The conditions that the cost function $c$ satisfies are very general. For example, if $c(p)=\infty$ for all measures $p \neq q$, then (7) reduces to (5), that is, preferences are expected utility. Similarly, setting $c(p)=0$ for all measures $p$ in a closed and convex set $C$ and $c(p)=\infty$ otherwise, denoted $c=\delta_{C}$, reduces (7) to the representation of the maxmin expected utility preferences of Gilboa and Schmeidler (1989).

As mentioned before, multiplier preferences also are a special case of variational preferences. They can be obtained by setting $c(p)=\theta R(p \| q)$. The next section shows that pinning down this functional form is possible with Savage's P2 applied to all Anscombe-Aumann acts. ${ }^{8}$

\footnotetext{
${ }^{8}$ If the existence of certainty equivalents of lotteries is assumed, that is, for any $\pi \in \Delta(Z)$ there exists $z \in Z$ with $z \sim \pi$, then $\mathrm{P} 2$ can be weakened and imposed only on purely subjective acts.
} 


\subsection{Axiomatization of Multiplier Preferences}

AXIOM P2-Savage's Sure-Thing Principle: For all $E \in \Sigma$ and $f, g, h, h^{\prime} \in$ $\mathcal{F}(\Delta(Z))$,

$$
f_{E} h \succsim g_{E} h \quad \Rightarrow \quad f_{E} h^{\prime} \succsim g_{E} h^{\prime} .
$$

DEFINITION 3: An event $E \in \Sigma$ is nonnull if there exist $f, g, h \in \mathcal{F}(\Delta(Z))$ such that $f E h \succ g E h$.

THEOREM 1: If $S$ has at least three disjoint nonnull events, then Axioms A1$\mathrm{A} 6, \mathrm{~A} 8$, and $\mathrm{P} 2$, are necessary and sufficient for $\succsim$ to have a multiplier representation (2). Moreover, in this case, two triples $\left(\theta^{\prime}, u^{\prime}, q^{\prime}\right)$ and $\left(\theta^{\prime \prime}, u^{\prime \prime}, q^{\prime \prime}\right)$ represent the same multiplier preference $\succsim$ if and only if $q^{\prime}$ and $q^{\prime \prime}$ are identical, and there exist $\alpha>0$ and $\beta \in \mathbb{R}$ such that $u^{\prime}=\alpha u^{\prime \prime}+\beta$ and $\theta^{\prime}=\alpha \theta^{\prime \prime}$.

The two cases $\theta=\infty$ (lack of concern for model misspecification) and $\theta<\infty$ (concern for model misspecification) can be distinguished on the basis of the independence axiom. ${ }^{9}$ In the case when $\theta$ is finite, its numerical value is uniquely determined, given $u$. A positive affine transformation of $u$ changes the scale on which $\theta$ operates, so $\theta$ has to change accordingly. This is reminiscent of the necessary adjustments of the constant absolute risk aversion coefficient when units of account are changed.

In addition, it should be mentioned that there exists an axiomatization by Wang (2003) of a class of preferences that includes multiplier preferences as a special case. However, his result is formally unrelated and it assumes different primitives: preferences are defined on triples $(f, C, q)$, where $f$ is an act with monetary payoffs, $C \subseteq \Delta(S)$ is a set of probability measures, and $q \in \Delta(S)$ is a reference measure. In particular, his axioms impose consistency conditions as the elements $C$ and $q$ are varied exogenously. In contrast, here $C=\Delta(S)$, and $q$ is fixed and derived from preferences.

\subsection{Sketch of the Proof}

The following variational formula (see, e.g., Proposition 1.4.2 of Dupuis and Ellis (1997)) plays a critical role in the analysis of multiplier preferences. For any bounded and $\Sigma$-measurable function $\xi: S \rightarrow \mathbb{R}$ and $q \in \Delta^{\sigma}(S)$,

$$
\min _{p \in \Delta S} \int_{S} \xi d p+\theta R(p \| q)=\phi_{\theta}^{-1}\left(\int_{S} \phi_{\theta} \circ \xi d q\right) .
$$

\footnotetext{
${ }^{9}$ The weaker certainty independence axiom is also sufficient for making such a distinction. Alternatively, Machina and Schmeidler's (1995) axiom of horse/roulette replacement or Grant and Polak's (2006) axiom of betting neutrality could be used.
} 
This formula implies that the variational

$$
\min _{p \in \Delta S} \int_{S} u(f(s)) d p+\theta R(p \| q)
$$

and the SOEU

$$
\int_{S} \phi_{\theta}(u(f(s))) d q(s)
$$

representations are ordinally equivalent, establishing the necessity of the MMR and the $\mathrm{P} 2$ axiom of Savage. ${ }^{10}$

The sufficiency argument relies on the fact that the MMR axioms guarantee the existence of an affine utility function $u: \Delta(Z) \rightarrow \mathbb{R}$ and a functional $I$ that maps utility-valued acts to reals, such that the functional $f \mapsto I(u(f))$ represents the preferences. For simplicity, assume that $u(\Delta(Z))=\mathbb{R}$. MMR showed that the functional $I$ has the translation invariance property that $I(u \circ f+k)=$ $I(u \circ f)+k$ for any act $f$ and any $k \in \mathbb{R}$. On the other hand, Axiom P2 together with the MMR axioms implies that the preferences have a SOEU representation

$$
\int_{S} \phi(u(f(s))) d q(s)
$$

for some strictly increasing function $\phi .{ }^{11}$ Moreover, Axioms A3 and A5 imply that $\phi$ is continuous and concave, and Axiom A8 implies that $q$ is countably additive. This representation, together with translation invariance, implies that

$$
\int_{S} \phi(u(f(s))) d q(s) \geq \int_{S} \phi(u(g(s))) d q(s)
$$

if and only if (iff)

$$
\int_{S} \phi(u(f(s))+k) d q(s) \geq \int_{S} \phi(u(g(s))+k) d q(s),
$$

which by uniqueness of the SOEU representation implies that there exist $\alpha(k)>0$ and $\beta(k) \in \mathbb{R}$ such that $\phi(x+k)=\alpha(k) \phi(x)+\beta(k)$ for all $x, k \in \mathbb{R}$.

\footnotetext{
${ }^{10}$ The fact that multiplier preferences rank purely subjective acts according to the EU criterion has been observed before in various levels of generality by Jacobson (1973), Whittle (1981), Skiadas (2003), and Maccheroni, Marinacci, and Rustichini (2006b). Because of this fact, it is not possible to distinguish multiplier preferences from the EU preferences based on the preferences over purely subjective acts alone: a setting with multiple sources of uncertainty, like the Anscombe-Aumann or Ergin-Gul ones, is needed.

${ }^{11}$ Recall that Savage's $\mathrm{P} 2$ axiom is imposed on $\mathcal{F}(\Delta(Z))$, not just the purely subjective acts, as in Savage (1972). The fact that imposing all Savage's axioms on $\mathcal{F}(\Delta(Z))$ implies SOEU was first shown by Neilson (1993). I am grateful to Peter Klibanoff for this reference.
} 
This is a generalized Pexider equation, whose only solutions are $\phi=\phi_{\theta}$ for $\theta \in(0, \infty]$, which establishes the sufficiency of the axioms.

\subsection{Discussion}

\subsubsection{Second-Order Expected Utility}

It follows from the above proof that the class of multiplier preferences is precisely the intersection of the class of variational preferences and the class of SOEU preferences. When viewed as a SOEU preference, multiplier preferences impose the condition $\phi=\phi_{\theta}$. When viewed as a variational preference, multiplier preferences impose the condition $c(p)=\theta R(p \| q)$. It is worthwhile to notice that this means that no other variational preferences have a SOEU representation, that is, assuming that $c$ is a statistical distance other than the relative entropy leads to models which do not have a SOEU representation for any $\phi$. Conversely, no other SOEU preference has a variational representation, that is, assuming that $\phi$ is a function other than the negative exponential leads to models which do not have a variational representation for any $c$.

\subsubsection{Source Sensitivity of Multiplier Preferences}

Focus on the case $\theta<\infty$ and notice that from the SOEU representation (9b), it follows that, for any two purely objective lotteries, $\pi^{\prime} \succsim \pi$ if and only if

$$
\sum_{z \in Z} u(z) \pi^{\prime}(z) \geq \sum_{z \in Z} u(z) \pi(z) .
$$

On the other hand, for any two purely subjective acts, $f^{\prime} \succsim f$ if and only if

$$
\sum_{z \in Z} \phi_{\theta}(u(z)) \pi_{f^{\prime}}(z) \geq \sum_{z \in Z} \phi_{\theta}(u(z)) \pi_{f}(z) .
$$

This means that the decision maker has a different attitude toward objective lotteries and toward subjective acts, while behaving according to EU in each subdomain. In particular, he is more averse toward subjective uncertainty (as captured by $\phi_{\theta} \circ u$ ) than toward objective risk (as captured by $u$ ). This phenomenon is called second-order risk aversion. ${ }^{12}$ What lead to the Ellsberg-type behavior are violations of EU across those domains. The following example shows that, because of this property, multiplier preferences can be useful for modeling Ellsberg-type behavior.

EXAMPLE 2-Ellsberg's Paradox Revisited: In the context of Example 1, consider a multiplier preference with a parameter $\theta$. Observe that $V\left(\pi_{R}\right)=$

\footnotetext{
${ }^{12}$ This notion was introduced by Ergin and Gul (2009) in a setting with two subjective sources of uncertainty (see Section 5).
} 
$V\left(\pi_{B}\right)=\phi_{\theta}\left(\frac{1}{2} u(100)+\frac{1}{2} u(0)\right)$, whereas $V\left(f_{R}\right)=q \phi_{\theta}(u(100))+(1-q) \times$ $\phi_{\theta}(u(0))$ and $V\left(f_{B}\right)=(1-q) \phi_{\theta}(u(100))+q \phi_{\theta}(u(0))$. Because of the indifference $V\left(f_{R}\right)=V\left(f_{B}\right)$, it follows that $q=\frac{1}{2}$; hence, $V\left(f_{R}\right)=V\left(f_{B}\right)=$ $\frac{1}{2} \phi_{\theta}(u(100))+\frac{1}{2} \phi_{\theta}(u(0))$. By Jensen's inequality, $\pi_{B} \sim \pi_{R} \succ f_{R} \sim f_{B}$ for all $\theta<\infty$. This means that the decision maker prefers objective risk to probabilistically equivalent subjective uncertainty, displaying behavior typical in Ellsberg's experiments.

\subsubsection{Measurement of Parameters}

Ellsberg's paradox provides a natural setting for the experimental measurement of the parameters of the model because the intensity of the preference for betting on the first versus the second urn, that is, the premium that the decision maker is willing to pay to switch between these two bets, is directly related to the value of the parameter $\theta$.

EXAMPLE 3: In the context of Examples 1 and 2, consider a multiplier preference with a constant relative risk aversion utility function $u(z)=(w+z)^{1-\gamma}$, where $w$ is the initial level of wealth, and parameter $\theta$. Observe that $V\left(\pi_{R}\right)=$ $V\left(\pi_{B}\right)=\phi_{\theta}\left(\frac{1}{2} w^{1-\gamma}+\frac{1}{2}(w+100)^{1-\gamma}\right)$, whereas $V\left(f_{R}\right)=V\left(f_{B}\right)=\frac{1}{2} \phi_{\theta}\left(w^{1-\gamma}\right)+$ $\frac{1}{2} \phi_{\theta}\left((w+100)^{1-\gamma}\right)$. Let $x$ denote the certainty equivalent of $\pi_{R}$ and $\pi_{B}$, that is, the amount of money that, when received for sure, would be indifferent to $\pi_{R}$ and $\pi_{B}$. Formally, $x$ solves

$$
(w+x)^{1-\gamma}=\frac{1}{2} w^{1-\gamma}+\frac{1}{2}(w+100)^{1-\gamma} .
$$

The observed value of the certainty equivalent $x$ allows computation of the curvature parameter $\gamma$ using Equation (11); let $\gamma(x)$ be the solution to this equation. ${ }^{13}$

Similarly, let $y$ be the certainty equivalent of $f_{R}$ and $f_{B}$, that is, the amount of money that, when received for sure, would be indifferent to $f_{R}$ and $f_{B}$. Formally, $y$ solves

$$
\phi_{\theta}\left((w+y)^{1-\gamma(x)}\right)=\frac{1}{2} \phi_{\theta}\left(w^{1-\gamma(x)}\right)+\frac{1}{2} \phi_{\theta}\left((w+100)^{1-\gamma(x)}\right) .
$$

The observed value of the certainty equivalent $y$ makes it possible to compute the parameter $\theta$ using equation (12); let $\theta(x, y)$ be the solution to this equation.

The procedure described above suggests that simple choice experiments could be used for empirical measurement of both $u$ and $\theta$. Such measurement

\footnotetext{
${ }^{13}$ If the utility function $u$ belongs to some higher-dimensional family of utility functions, more certainty equivalents need to be elicited so as to infer all of its parameters.
} 
of parameters would be very useful in applied settings, where it is important to know the numerical values of parameters. For example, the macro-finance literature devotes a lot of attention to the discrepancy between the micro- and macro-level estimates of the curvature of $u$. By analogy, it would be valuable to know the micro-level estimate of $\theta$ to be able to compare it to the value calibrated from the macro-level data. The above procedure provides a simple "revealed-preference" method of comparison that is complementary to the heuristic method of "detection error probabilities" developed by Anderson, Hansen, and Sargent (2003) and Hansen and Sargent (2007).

\section{AXIOMATIZATION WITH TWO SUBJECTIVE SOURCES}

This section discusses a choice domain that does not rely on the assumption of objective risk: instead, there are two sources of subjective uncertainty, toward which the decision maker may have different attitudes. This type of environment was discussed by Chew and Sagi (2008), Ergin and Gul (2009), Gul and Pesendorfer (2010), and Nau $(2001,2006)$.

\subsection{Subjective Sources of Uncertainty}

Assume that the state space has a product structure $S=S_{a} \times S_{b}$, where $a$ and $b$ are two separate issues, or sources of uncertainty, toward which the decision maker may have different attitudes. In comparison with the AnscombeAumann framework, where objective risk is one of the sources, here both sources are subjective. Let $\mathcal{A}_{a}$ be a sigma algebra of subsets of $S_{a}$ and let $\mathcal{A}_{b}$ be a sigma algebra of subsets of $S_{b}$. Let $\Sigma_{a}$ be the sigma algebra of sets of the form $A \times S_{b}$ for all $A \in \mathcal{A}_{a}$, let $\Sigma_{b}$ be the sigma algebra of sets of the form $S_{a} \times B$ for all $B \in \mathcal{A}_{b}$, and let $\Sigma$ be the sigma algebra generated by $\Sigma_{a} \cup \Sigma_{b}$. Let $\mathcal{F}(Z)$ be the set of all simple $\Sigma$-measurable acts $f: S \rightarrow Z$; moreover, let $\mathcal{F}_{a}(Z)$ be the set of acts that are $\Sigma_{a}$-measurable, and likewise for $\mathcal{F}_{b}(Z)$. Let $\mathcal{U}$ denote the set $u(Z)$.

Ergin and Gul (2009) axiomatized preferences which are general enough to accommodate probabilistic sophistication and even second-order probabilistic sophistication. An important subclass of those preferences are second-order expected utility preferences represented by

$$
V(f)=\int_{S_{b}} \phi\left(\int_{S_{a}} u\left(f\left(s_{a}, s_{b}\right)\right) d q_{a}\left(s_{a}\right)\right) d q_{b}\left(s_{b}\right),
$$

where the measures $q_{a} \in \Delta\left(S_{a}\right)$ and $q_{b} \in \Delta\left(S_{b}\right)$ are convex-ranged, ${ }^{14} u: Z \rightarrow \mathbb{R}$, and $\phi: D_{\phi} \rightarrow \mathbb{R}$ is a strictly increasing and continuous function with domain

\footnotetext{
${ }^{14} \mathrm{~A}$ measure $q$ is convex-ranged if for every $E \in \Sigma$ and every $\alpha \in(0,1)$ there exists $\Sigma \ni E^{\prime} \subseteq E$ with $q\left(E^{\prime}\right)=\alpha q(E)$. It is well known that this requirement is equivalent to nonatomicity for $q \in \Delta^{\sigma}(S)$. Any measure on $\mathbb{R}$ that has a density with respect to the Lebesgue measure has this property; in applications of multiplier preferences, $q$ is most often a Normal distribution.
} 
$D_{\phi}:=\left\{\int_{S_{a}} u\left(f\left(s_{a}, s_{b}\right)\right) d q_{a}\left(s_{a}\right) \mid f \in \mathcal{F}_{a}(Z)\right\}$. To characterize preferences represented by (13), Ergin and Gul (2009) used the following axioms.

AXIOM P1'-Weak Order: The preference $\succsim$ is complete and transitive.

AxIOM P2'-Sure-Thing Principle: For all events $E_{a} \in \Sigma_{a}$ and $E_{b} \in \Sigma_{b}$, and acts $f, g, h, h^{\prime} \in \mathcal{F}_{a}(Z)$ and $\tilde{f}, \tilde{g}, \tilde{h}, \tilde{h}^{\prime} \in \mathcal{F}(Z)$, the following relationships exist:

(a) $f_{E_{a}} h \succsim g_{E_{a}} h$ iff $f_{E_{a}} h^{\prime} \succsim g_{E_{a}} h^{\prime}$.

(b) $\tilde{f}_{E_{b}} \tilde{h} \succsim \tilde{g}_{E_{b}} \tilde{h}$ iff $\tilde{f}_{E_{b}} \tilde{h}^{\prime} \succsim \tilde{g}_{E_{b}} \tilde{h}^{\prime}$.

Axiom P3'-Eventwise Monotonicity: For all $z, z^{\prime} \in Z, f \in \mathcal{F}(Z)$, and all nonnull events $E \in \Sigma, z_{E} f \succsim z_{E}^{\prime} f$ iff $z \succsim z^{\prime}$.

AxıOM P4-Weak Comparative Probability:

(a) For all $x \succ y$ and $x^{\prime} \succ y^{\prime}$, and $A, A^{\prime} \in \Sigma_{A}, x_{A} y \succ x_{A^{\prime}} y$ iff $x_{A}^{\prime} y^{\prime} \succ x_{A^{\prime}}^{\prime} y^{\prime}$.

(b) For all $f, g, f^{\prime}, g^{\prime} \in \mathcal{F}_{a}(Z)$ such that $f \succ g$ and $f^{\prime} \succ g^{\prime}$, and $B, B^{\prime} \in \Sigma_{b}$, $f_{B} g \succ f_{B^{\prime}} g$ iff $f_{B}^{\prime} g^{\prime} \succ f_{B^{\prime}}^{\prime} g^{\prime}$.

AXIOM P5'-Nondegeneracy: There exist $x, y \in Z$ such that $x \succ y$.

AXIOM P6-Small Event Continuity: For all $f, g \in \mathcal{F}(Z)$ with $f \succ g$, and all $z \in Z$, the following statements hold:

(a) There exists a partition $E_{1}, \ldots, E_{n} \in \Sigma_{a}$ of $S$ such that for all $i, z_{E_{i}} f \succ g$ and $f \succ z_{E_{i}}$ g.

(b) There exists a partition $F_{1}, \ldots, F_{m} \in \Sigma_{b}$ of $S$ such that for all $j, z_{F_{j}} f \succ g$ and $f \succ z_{F_{j}}$ g.

There is a close relationship between representations (13) and (10). The role of objective risk is now taken by a subjective source: issue $a$. For each $s_{b}$, the decision maker computes the expected utility of $f\left(\cdot, s_{b}\right)$ and then averages those values using function $\phi$.

\subsection{Second-Order Risk Aversion}

In the Anscombe-Aumann framework, the concavity of the function $\phi$ is responsible for second-order risk aversion, that is, higher aversion toward subjective uncertainty than toward objective risk. This property is a consequence of the axiom of uncertainty aversion (Axiom A5). Similarly, in the present setup, the concavity of the function $\phi$ is responsible for higher aversion toward issue $b$ than toward issue $a$. This property was introduced by Ergin and Gul (2009), who formally defined it in terms of mean-preserving spreads. However, this 
definition refers to the probability measures obtained from the representation and hence is not expressed directly in terms of observables. ${ }^{15}$

In the presence of other axioms, the following purely behavioral axiom is equivalent to Ergin and Gul's (2009) definition.

AXIOM A5'-Second-Order Risk Aversion: For any $f, g \in \mathcal{F}_{b}(Z)$ and any $E \in \Sigma_{a}$, if $f \sim g$, then $f_{E} g \succsim f$.

This axiom is a direct subjective analog of Schmeidler's (1989) axiom of uncertainty aversion (Axiom A5).

THEOREM 2: Suppose $\succsim$ satisfies Axioms $\mathrm{P} 1^{\prime}-\mathrm{P} 6{ }^{\prime}$ and certainty equivalents exist, that is, for any $f \in \mathcal{F}(Z)$, there exists $z \in Z$ with $z \sim f$. Then Axiom A5' is satisfied if and only if the function $\phi$ in (13) is concave. ${ }^{16}$

\subsection{Axiomatization of Multiplier Preferences}

The additional axiom that delivers multiplier preferences in this framework is weak $\mathcal{F}_{a}$ independence, which is the direct subjective analog of the weak certainty independence axiom (Axiom A2) of MMR.

AXIOM A2'-Weak $\mathcal{F}_{a}$ Independence: For any event $E \in \Sigma_{a}$ for all $f, g \in$ $\mathcal{F}(Z)$ and $h, h^{\prime} \in \mathcal{F}_{a}(Z)$,

$$
f_{E} h \succsim g_{E} h \quad \Rightarrow \quad f_{E} h^{\prime} \succsim g_{E} h^{\prime} .
$$

In addition, a technical axiom, similar to Axiom A8, is needed.

AXIOM A8'- $\mathcal{F}_{b}$-Monotone Continuity: If $f, g \in \mathcal{F}(Z), x \in Z,\left\{E_{n}\right\}_{n \geq 1} \in \Sigma_{b}$ with $E_{1} \supseteq E_{2} \supseteq \cdots$, and $\bigcap_{n \geq 1} E_{n}=\emptyset$, then $f \succ g$ implies that there exists $N \geq 1$ such that $x_{E_{N}} f \succ g$.

THEOREM 3: Axioms $\mathrm{P} 1^{\prime}-\mathrm{P} 6{ }^{\prime}, \mathrm{A} 2^{\prime}, \mathrm{A} 5^{\prime}$, and $\mathrm{A} 8^{\prime}$ are necessary and sufficient for $\succsim$ to be represented by $V$, where

$$
V(f)=\min _{p_{b} \in \Delta\left(S_{b}\right)} \int_{S_{b}}\left(\int_{S_{a}} u\left(f\left(s_{a}, s_{b}\right)\right) d q_{a}\left(s_{a}\right)\right) d p_{b}\left(s_{b}\right)+\theta R\left(p_{b} \| q_{b}\right)
$$

and $u: Z \rightarrow \mathbb{R}, \theta \in(0, \infty]$, and $q_{a} \in \Delta\left(S_{a}\right), q_{b} \in \Delta^{\sigma}\left(S_{b}\right)$ are convex-ranged measures.

${ }^{15}$ Theorems 2 and 5 of Ergin and Gul (2009) characterize second-order risk aversion in terms of induced preferences over induced Anscombe-Aumann acts and an analog of Axiom A5 in that induced setting. However, just as with mean-preserving spreads, those induced AnscombeAumann acts are constructed using the subjective probability measure derived from the representation.

${ }^{16}$ The full analysis that does not rely on the existence of certainty equivalents is contained in Appendix A.2. 


\subsection{Sketch of the Proof}

By Theorem 3 of Ergin and Gul (2009), Axioms P1'-P6' are equivalent to $\succsim$ being represented by (13). Assume for simplicity that certainty equivalents exist and that $u(Z)=\mathbb{R}$. By Theorem 2 , Axiom $A 5^{\prime}$ is equivalent to $\phi: \mathbb{R} \rightarrow \mathbb{R}$ being concave. Moreover, it is easy to see that Axiom A8' is equivalent to $q_{b}$ being countably additive. A direct verification establishes that Axiom A2' is necessary.

To establish the sufficiency of Axiom A2', observe that by the convexrangedness of $q_{a}$, there exists an event $E \in \Sigma_{a}$ such that $q_{a}(E)=\frac{1}{2}$. For all $k \in \mathbb{R}$ and $f^{\prime}, g^{\prime} \in \mathcal{F}_{b}(Z)$, Axiom A2' (applied to $f, g, h$, and $h^{\prime}$ such that $u(f)=2 u\left(f^{\prime}\right), u(g)=2 u\left(g^{\prime}\right), u(h)=0$, and $\left.u\left(h^{\prime}\right)=2 k\right)$ implies that

$$
\int_{S_{b}} \phi\left(u\left(f^{\prime}\left(s_{b}\right)\right)\right) d q_{b}\left(s_{b}\right) \geq \int_{S_{b}} \phi\left(u^{\prime}\left(g\left(s_{b}\right)\right)\right) d q_{b}\left(s_{b}\right)
$$

iff

$$
\int_{S_{b}} \phi\left(u\left(f^{\prime}\left(s_{b}\right)\right)+k\right) d q_{b}\left(s_{b}\right) \geq \int_{S_{b}} \phi\left(u\left(g^{\prime}\left(s_{b}\right)\right)+k\right) d q_{b}\left(s_{b}\right),
$$

which, by the same argument as in the proof of Theorem 1, implies that $\phi=\phi_{\theta}$.

\section{CONCLUSION}

One of the challenges in decision theory lies in finding decision models that would do better than expected utility in describing individual choices, but would at the same time be easy to incorporate into economic models of aggregate behavior.

This paper studies the model of multiplier preferences which is known to satisfy the latter requirement. By obtaining an axiomatic characterization of this model, the paper studies its individual choice properties, which will help to determine whether it also satisfies the first requirement mentioned above.

The axiomatization provides a set of testable implications of the model, which will be helpful in its empirical verification. The axiomatization also enables measurement of the parameters of the model on the basis of observable choice data alone, thereby providing a useful tool for applications of the model.

\section{APPENDIX: PROOFS}

Let $B_{0}(\Sigma)$ denote the set of all real-valued $\Sigma$-measurable simple functions and let $B_{0}(\Sigma, K)$ be the set of all functions in $B_{0}(\Sigma)$ that take values in a convex set $K \subseteq \mathbb{R}$.

In the course of the proof of Theorem 1, a result of Grant, Polak, and Strzalecki (2009) will be invoked that delivers a SOEU representation on each finite 
partition of $S$. The following theorem shows that these representations can be "patched" together to obtain an overall SOEU representation on $S$.

Let $\Xi$ denote the set of all finite partitions of $S$ that are composed of events in $\Sigma$; let $\Xi^{3} \subseteq \Xi$ denote the set of all such partitions that contain at least three nonnull events. For any $\mathcal{G} \in \Xi$, let $\mathcal{A}(\mathcal{G})$ be the algebra generated by $\mathcal{G}$. For any $\mathcal{G} \in \Xi^{3}$, let $\mathcal{F}_{\mathcal{G}}(\Delta(Z))$ denote the set of acts in $\mathcal{F}(\Delta(Z))$ that are measurable with respect to $\mathcal{A}(\mathcal{G})$.

DEFINITION $4-\Xi^{3}$-SOEU: A preference $\succsim$ on $\mathcal{F}(\Delta(Z))$ is $\Xi^{3}$-SOEU iff for any $\mathcal{G} \in \Xi^{3}$, the restriction of $\succsim$ to $\mathcal{F}_{\mathcal{G}}(\Delta(Z))$ is represented by $f \mapsto$ $\sum_{E \in \mathcal{G}} \phi_{\mathcal{G}}\left(u_{\mathcal{G}}(f(E))\right) p_{\mathcal{G}}(E)$ with a nonconstant affine function $u_{\mathcal{G}}: \Delta(Z) \rightarrow$ $\mathbb{R}$ with range $\mathcal{U}_{\mathcal{G}}$, a strictly increasing, continuous, and concave function $\phi_{\mathcal{G}}: \mathcal{U}_{\mathcal{G}} \rightarrow \mathbb{R}$ and measure $p_{\mathcal{G}}: \mathcal{A}(\mathcal{G}) \rightarrow[0,1]$ such that at least three events in $\mathcal{G}$ have nonzero probability.

THEOREM 4: Suppose that $\Xi^{3} \neq \varnothing$ and that $\succsim$ satisfies Axioms A1, A4, and $\mathrm{P} 2$. The preference $\succsim$ is $\Xi^{3}-S O E U$ if and only if there exists a measure $p \in \Delta(S)$ and a nonconstant affine function $u: \Delta(Z) \rightarrow \mathbb{R}$ with range $\mathcal{U}$, and a strictly increasing, concave, and continuous function $\phi: \mathcal{U} \rightarrow \mathbb{R}$ such that $\succsim$ is represented by $f \mapsto \int_{S} \phi(u(f(s))) d p(s)$.

PROOF: The sufficiency of the representation is straightforward. For necessity, let $\mathcal{G}, \mathcal{G}^{\prime} \in \Xi^{3}$. The restrictions of $\succsim$ to $\mathcal{F}_{\mathcal{G}}(\Delta(Z))$ and to $\mathcal{F}_{\mathcal{G}^{\prime}}(\Delta(Z))$ coincide on constant acts $\Delta(Z)$. Thus, from the uniqueness of the von NeumannMorgenstern utility, it follows that $u_{\mathcal{G}}$ and $u_{\mathcal{G}^{\prime}}$ are identical up to a positive affine transformation. Fix any two prizes $z \succ z^{\prime}$ and for each $\mathcal{G} \in \Xi^{3}$, normalize $u_{\mathcal{G}}$ so that $u_{\mathcal{G}}(z)=1$ and $u_{\mathcal{G}}\left(z^{\prime}\right)=0$. Define $u$ to be the common utility function for all $\mathcal{G} \in \Xi^{3}$.

For any $\mathcal{G}, \mathcal{G}^{\prime} \in \Xi$, define $\mathcal{G} \geq \mathcal{G}^{\prime}$ iff $\mathcal{G}$ is finer than $\mathcal{G}^{\prime}$, that is, for every $E \in \mathcal{G}$ there exists $F \in \mathcal{G}^{\prime}$ with $E \subseteq F$. For any $\mathcal{G}, \mathcal{G}^{\prime} \in \Xi$, let $\mathcal{G} \vee \mathcal{G}^{\prime}$ be their coarsest common refinement and let $\mathcal{G} \wedge \mathcal{G}^{\prime}$ be their finest common coarsening.

LEMMA 1: If $E \in \Sigma$ is nonnull, then for any finite $\Sigma$-measurable partition $\left\{F_{1}, \ldots, F_{n}\right\}$ of $E$, at least one of the sets $F_{1}, \ldots, F_{n}$ is nonnull.

\section{PROOF:}

CLAIM 1: For any nonnull $E \in \Sigma$, there exist $\pi, \rho, \sigma \in \Delta(Z)$ such that $\rho_{E} \sigma \succ$ $\pi_{E} \sigma$.

ProOF: There exist $f, g, h \in \mathcal{F}(\Delta(Z))$ such that $f_{E} h \succ g_{E} h$. By P2 choose $h$ to equal to some $\sigma \in \Delta(Z)$ different than any of the prizes given by $f$ and $g$. Let $\left\{E_{1}, \ldots, E_{n}, E^{c}\right\} \in \Xi$ be a partition of $S$ with respect to which both $f_{E} \sigma$ and $g_{E} \sigma$ are measurable. Let $\rho$ be the most preferred element among $\left\{f\left(E_{i}\right) \mid i=\right.$ 
$1, \ldots, n\}$ and let $\pi$ be the least preferred element among $\left\{g\left(E_{i}\right) \mid i=1, \ldots, n\right\}$. By A4, $\rho_{E} \sigma \succsim f_{E} \sigma$ and $g_{E} \sigma \succsim \pi_{E} \sigma$. Thus $\rho_{E} \sigma \succ \pi_{E} \sigma$.

CLAIM 2: For any nonnull $E \in \Sigma$ and any two-element $\Sigma$-measurable partition $\left\{F_{1}, F_{2}\right\}$ of $E$, at least one of the sets $F_{1}, F_{2}$ is nonnull.

PROOF: Suppose that there exists a two-element $\Sigma$-measurable partition $\left\{F_{1}, F_{2}\right\}$ of $E$ such that both sets $F_{1}$ and $F_{2}$ are null. Then $\rho_{F_{i}} h \sim \pi_{F_{i}} h$ for any $h \in \mathcal{F}(\Delta(Z))$ and all $i=1,2$. In particular, $\rho_{E} \sigma=\rho_{F_{1}}\left(\rho_{F_{2}} \sigma\right) \sim \pi_{F_{1}}\left(\rho_{F_{2}} \sigma\right)=$ $\rho_{F_{2}}\left(\pi_{F_{1}} \sigma\right) \sim \pi_{F_{2}}\left(\pi_{F_{1}} \sigma\right)=\pi_{E} \sigma$. This is a contradiction with Claim 1. Q.E.D.

CLAIM 3-Inductive Step: If for any nonnull $E^{\prime} \in \Sigma$ and any n-element $\Sigma$ measurable partition $\left\{F_{1}, \ldots, F_{n}\right\}$ of $E^{\prime}$, at least one of the sets $F_{1}, \ldots, F_{n}$ is nonnull, then for any nonnull $E \in \Sigma$ and any $n+1$-element $\Sigma$-measurable partition $\left\{F_{1}, \ldots, F_{n+1}\right\}$ of $E$, at least one of the sets $F_{1}, \ldots, F_{n+1}$ is nonnull.

Proof: By Claim 2, at least one of the sets $F_{1} \cup \cdots \cup F_{n}=: E^{\prime}$ and $F_{n+1}$ is nonnull. If the latter is true, this concludes the proof. If $E^{\prime}$ is nonnull, then the premise of this claim applied to the set $E^{\prime}$ and its partition $\left\{F_{1}, \ldots, F_{n}\right\}$ concludes the proof.

Q.E.D.

LEMMA 2: $\mathcal{G} \vee \mathcal{G}^{\prime} \in \Xi^{3}$ for any $\mathcal{G} \in \Xi^{3}$ and $\mathcal{G}^{\prime} \in \Xi$.

PROOF: By assumption, there are at least three disjoint nonnull sets in $\mathcal{G}$. By Lemma 1 for any such set $E \in \mathcal{G}$, there is at least one nonnull member of $\{E \cap$ $F \mid F \in \mathcal{G}^{\prime}$ \}. Thus, there are at least three nonnull members of $\mathcal{G} \vee \mathcal{G}^{\prime}$. Q.E.D.

LEMMA 3: The functions $\left\{\phi_{\mathcal{G}}\right\}_{\mathcal{G} \in \Xi^{3}}$ and measures $\left\{p_{\mathcal{G}}\right\}_{\mathcal{G} \in \Xi^{3}}$ can be chosen in such a way that there exists $\phi: \mathcal{U} \rightarrow \mathbb{R}$ such that $\phi_{\mathcal{G}}=\phi$ for any $\mathcal{G} \in \Xi^{3}$, and for any $\mathcal{G}, \mathcal{G}^{\prime} \in \Xi^{3}$, the restrictions of measures $p_{\mathcal{G}}$ and $p_{\mathcal{G}^{\prime}}$ to $\mathcal{A}\left(\mathcal{G} \wedge \mathcal{G}^{\prime}\right)$ coincide.

ProOF: For each $\mathcal{G} \in \Xi^{3}$, normalize $\phi_{\mathcal{G}}$ so that $\phi_{\mathcal{G}}(u(z))=1$ and $\phi_{\mathcal{G}}\left(u\left(z^{\prime}\right)\right)=0$. First, let $\mathcal{G}, \mathcal{H} \in \Xi^{3}$ such that $\mathcal{G} \geq \mathcal{H}$. Observe, that $\mathcal{F}_{\mathcal{H}}(\Delta(Z)) \subseteq$ $\mathcal{F}_{G}(\Delta(Z))$, so both $\left(\phi_{\mathcal{G}}, p_{\mathcal{G}}\right)$ and $\left(\phi_{\mathcal{H}}, p_{\mathcal{H}}\right)$ represent preferences on $\mathcal{F}_{\mathcal{H}}(\Delta(Z))$. By the uniqueness of the expected utility representation, the restriction of $p_{\mathcal{H}}$ to $\mathcal{G}$ coincides with $p_{\mathcal{G}}$ and the functions $\phi_{\mathcal{H}}$ and $\phi_{\mathcal{G}}$ are identical up to a positive affine transformation, which by the above normalization assumption implies that they are equal.

Second, let $\mathcal{G}, \mathcal{G}^{\prime} \in \Xi^{3}$. By Lemma $2, \mathcal{G} \vee \mathcal{G}^{\prime} \in \Xi^{3}$. Furthermore, $\mathcal{G} \vee \mathcal{G}^{\prime} \geq$ $\mathcal{G}$ and $\mathcal{G} \vee \mathcal{G}^{\prime} \geq \mathcal{G}^{\prime}$. From the above paragraph, it follows that $\phi_{\mathcal{G}}=\phi_{\mathcal{G} \vee \mathcal{G}^{\prime}}=$ $\phi_{\mathcal{G}^{\prime}}$. Let $\phi$ be this common function. Also the restriction of $p_{\mathcal{G} \vee \mathcal{G}^{\prime}}$ to $\mathcal{A}(\mathcal{G})$ coincides with $p_{\mathcal{G}}$; hence the restriction of $p_{\mathcal{G} \vee \mathcal{G}^{\prime}}$ to $\mathcal{A}\left(\mathcal{G} \wedge \mathcal{G}^{\prime}\right)$ coincides with the restriction of $p_{\mathcal{G}}$ to $\mathcal{A}\left(\mathcal{G} \wedge \mathcal{G}^{\prime}\right)$. Likewise, the restriction of $p_{\mathcal{G} \vee \mathcal{G}^{\prime}}$ to $\mathcal{A}\left(\mathcal{G}^{\prime}\right)$ coincides with $p_{\mathcal{G}^{\prime}}$; hence the restriction of $p_{\mathcal{G} \vee \mathcal{G}^{\prime}}$ to $\mathcal{A}\left(\mathcal{G} \wedge \mathcal{G}^{\prime}\right)$ coincides with the restriction of $p_{\mathcal{G}^{\prime}}$ to $\mathcal{A}\left(\mathcal{G} \wedge \mathcal{G}^{\prime}\right)$.

Q.E.D. 
LEMMA 4: There exists $p \in \Delta(S)$ such that $p(E)=p_{\mathcal{H}}(E)$ for any $E \in \Sigma$ and any $\mathcal{H} \in \Xi^{3}$ with $E \in \mathcal{H}$.

ProOF: Let $\overline{\mathcal{G}} \in \Xi^{3}$ be some fixed element of $\Xi^{3}$. For any $E \in \Sigma$, define the partition $\mathcal{G}_{E}:=\overline{\mathcal{G}} \vee\left\{E, E^{c}\right\}$; by Lemma $2, \mathcal{G}_{E} \in \Xi^{3}$. Define the function $p: \Sigma \rightarrow$ $[0,1]$ by $p(E):=p_{\mathcal{G}_{E}}(E)$. Let $E, F \in \Sigma$ be such that $E \cap F=\emptyset$. Let $\mathcal{G}_{E F}:=\overline{\mathcal{G}} \vee$ $\left\{E, F,(E \cup F)^{c}\right\}$. By Lemma $2, \mathcal{G}_{E F} \in \Xi^{3}$ and by Lemma $3, p_{\mathcal{G}_{E}}(E)=p_{\mathcal{G}_{E F}}(E)$ because $E \in \mathcal{A}\left(\mathcal{G}_{E} \wedge \mathcal{G}_{E F}\right)$. Likewise, $p_{\mathcal{G}_{F}}(F)=p_{\mathcal{G}_{E F}}(F)$ because $F \in \mathcal{A}\left(\mathcal{G}_{F} \wedge\right.$ $\left.\mathcal{G}_{E F}\right)$. Also, $p_{\mathcal{G}_{E \cup F}}(E \cup F)=p_{\mathcal{G}_{E F}}(E \cup F)$ because $E \cup F \in \mathcal{A}\left(\mathcal{G}_{E \cup F} \wedge \mathcal{G}_{E F}\right)$. By definition, $p(E \cup F)=p_{\mathcal{G}_{E \cup F}}(E \cup F)=p_{\mathcal{G}_{E F}}(E \cup F)=p_{\mathcal{G}_{E F}}(E)+p_{\mathcal{G}_{E F}}(F)=$ $p_{\mathcal{G}_{E}}(E)+p_{\mathcal{G}_{F}}(F)=p(E)+p(F)$. Hence, $p \in \Delta(S)$.

Suppose $\mathcal{H} \in \Xi^{3}$ and $E \in \mathcal{H}$. Then $E \in \mathcal{A}\left(\mathcal{H} \wedge \mathcal{G}_{E}\right)$ and by Lemma 3, $p_{\mathcal{H}}(E)=p_{\mathcal{G}_{E}}(E)$. Hence, by definition, $p(E)=p_{\mathcal{H}}(E)$.

Q.E.D.

Conclusion of THE Proof of TheOREM 4: For any act $f \in \mathcal{F}(\Delta(Z))$, define $V(f):=\int \phi(u(f(s)) d p(s)$. To verify that $V$ represents $\succsim$, let $f, g \in$ $\mathcal{F}(\Delta(Z))$. Let $\mathcal{G}_{f g} \in \Xi$ be a partition such that both $f$ and $g$ are measurable with respect to $\mathcal{A}\left(\mathcal{G}_{f g}\right)$. Let $\overline{\mathcal{G}} \in \Xi^{3}$ be some fixed element of $\Xi^{3}$ and let $\mathcal{G}:=\mathcal{G}_{f g} \vee \overline{\mathcal{G}}$; by Lemma $2, \mathcal{G} \in \Xi^{3}$. By assumption, $f \succsim g$ iff $\sum_{E \in \mathcal{G}} \phi_{\mathcal{G}}\left(u_{\mathcal{G}}(f(E)) p_{\mathcal{G}}(E) \geq \sum_{E \in \mathcal{G}} \phi_{\mathcal{G}}\left(u_{\mathcal{G}}(g(E)) p_{\mathcal{G}}(E)\right.\right.$. Since $u_{\mathcal{G}}=u, \boldsymbol{\phi}_{\mathcal{G}}=\phi$ (by Lemma 3), and $p(E)=p_{\mathcal{G}}(E)$ for all $E \in \mathcal{G}$ (by Lemma 4), it follows that $f \succsim g$ iff $\sum_{E \in \mathcal{G}} \phi\left(u(f(E)) p(E) \geq \sum_{E \in \mathcal{G}} \phi(u(g(E)) p(E)\right.$.

Q.E.D.

\section{A.1. Proof of Theorem 1}

The necessity of the axioms was shown in the sketch of proof (Section 3.3); this section focuses on sufficiency. Uniqueness follows from Corollary 5 of MMR.

\section{A.1.1. Niveloidal Representation}

By Lemmas 25 and 28 of MMR and Lemma 22 in Maccheroni, Marinacci, and Rustichini (2004), Axioms A1-A6 imply that there exists a nonconstant affine function $u: \Delta(Z) \rightarrow \mathbb{R}$ and a concave functional $I: B_{0}(\Sigma, \mathcal{U}) \rightarrow \mathbb{R}$ such that $f \succsim g$ iff $I(u \circ f) \geq I(u \circ g)$, where $\mathcal{U}:=u(\Delta(Z))$. Moreover, within this class, $u$ is unique up to positive affine transformations.

Without loss of generality (wlog), $\mathcal{U} \in\left\{\mathbb{R}, \mathbb{R}_{+}, \mathbb{R}_{-},[0,1]\right\}$; the inclusion of the endpoints does not matter for further analysis. For any $y \in \operatorname{int} \mathcal{U}$, define $\mathcal{U}^{y}:=$ $\{x \in \mathcal{U} \mid x+y \in \mathcal{U}\}$. The functional $I$ has the property that $I(\xi+y)=I(\xi)+y$ for all $y \in \mathcal{U}$ and $\xi \in B_{0}\left(\Sigma, \mathcal{U}^{y}\right)$.

\section{A.1.2. Utility Acts}

For each act $f$, define the utility act associated with $f$ as $u \circ f \in B_{0}(\Sigma, \mathcal{U})$. The preference on acts induces a preference on utility acts: for any $\xi^{\prime}, \xi^{\prime \prime} \in$ 
$B_{0}(\Sigma, \mathcal{U})$, define $\xi^{\prime} \succsim_{u} \xi^{\prime \prime}$ iff $f^{\prime} \succsim f^{\prime \prime}$, for some $\xi^{\prime}=u \circ f^{\prime}$ and $\xi^{\prime \prime}=u \circ f^{\prime \prime}$. The choice of particular versions of $f^{\prime}$ and $f^{\prime \prime}$ is irrelevant, because of Axiom A4.

From Section A.1.1, it follows that $\xi^{\prime} \succsim_{u} \xi^{\prime \prime}$ iff $I\left(\xi^{\prime}\right) \geq I\left(\xi^{\prime \prime}\right)$ iff $I\left(\xi^{\prime}+y\right) \geq$ $I\left(\xi^{\prime \prime}+y\right)$ iff $\xi^{\prime}+y \succsim_{u} \xi^{\prime \prime}+y$ for all $y \in$ int $\mathcal{U}$ and all $\xi^{\prime}, \xi^{\prime \prime} \in B_{0}\left(\Sigma, \mathcal{U}^{y}\right)$.

\section{A.1.3. Second-Order Expected Utility}

By Proposition 7 of MMR the preference $\succsim$ is ambiguity averse in the sense of Ghirardato and Marinacci (2002). Observe that for any $\mathcal{G} \in \Xi^{3}$, there is a natural bijection between $\mathcal{F}_{\mathcal{G}}(\Delta(Z))$ and $(\Delta(Z))^{|\mathcal{G}|}$ that preserves Axioms A1-A6, P2, and the ambiguity aversion in the sense of Ghirardato and Marinacci (2002). Thus, by Theorem 2 of Grant, Polak, and Strzalecki (2009), for any $\mathcal{G} \in \Xi^{3}$, the restriction of $\succsim$ to $\mathcal{F}_{\mathcal{G}}(\Delta(Z))$ has an additive representation $f \mapsto \sum_{E \in \mathcal{G}} \phi_{\mathcal{G}}\left(u_{\mathcal{G}}(f(E))\right) p_{\mathcal{G}}(E)$ with a nonconstant affine function $u_{\mathcal{G}}: \Delta(Z) \rightarrow \mathbb{R}$ with range $\mathcal{U}_{\mathcal{G}}$, a strictly increasing, continuous, and concave function $\phi_{\mathcal{G}}: \mathcal{U}_{\mathcal{G}} \rightarrow \mathbb{R}$, and measure $p_{\mathcal{G}}: \mathcal{A}(\mathcal{G}) \rightarrow[0,1]$ such that at least three events in $\mathcal{G}$ have nonzero probability. By Theorem 4 above, the preference $\succsim$ on $\mathcal{F}(\Delta(Z))$ has an additive representation

$$
\int_{S} \phi(u(f(s))) d q(s),
$$

where the measure $q \in \Delta(S)$ and the function $\phi: \mathcal{U} \rightarrow \mathbb{R}$ is strictly increasing, concave, and continuous.

By Theorem 1 in Villegas (1964, Section 1), Axiom A8 implies that $q \in$ $\Delta^{\sigma}(S)$.

\section{A.1.4. Proof That $\phi=\phi_{\theta}$}

For any $y \in \operatorname{int} \mathcal{U}$, define $\phi^{y}(x):=\phi(x+y)$ for all $x \in \mathcal{U}^{y}$. It follows from Sections A.1.2 and A.1.3 that $\int_{S} \phi^{y} \circ \xi^{\prime} d q \geq \int_{S} \phi^{y} \circ \xi^{\prime \prime} d q$ iff $\int_{S} \phi \circ \xi^{\prime} d q \geq$ $\int_{S} \phi \circ \xi^{\prime \prime} d q$ for all $\xi^{\prime}, \xi^{\prime \prime} \in B_{0}\left(\Sigma, \mathcal{U}^{y}\right)$. Thus, $(\phi, q)$ and $\left(\phi^{y}, q\right)$ are EU representations of the same preference on $B_{0}\left(\Sigma, \mathcal{U}^{y}\right)$. By the uniqueness (up to positive affine transformation) of the EU representation, it follows that $\phi(x+y)=\alpha(y) \phi(x)+\beta(y)$ for all $y \in \operatorname{int} \mathcal{U}$ and all $x \in \mathcal{U}^{y}$. This is a generalization of Pexider's equation (see equation (3) of Aczél (1966, Section 3.1.3, p. 148)). If $\mathcal{U}$ is unbounded, then by Corollary 1 in Aczél (1966, Section 3.1.3), up to positive affine transformations, the only strictly increasing concave solutions are of the form $\phi_{\theta}$, for $\theta \in(0, \infty]$. If $\mathcal{U}$ is bounded, then wlog assume that int $\mathcal{U}=(0,1)$ and define the set $R:=\left\{(x, y) \in \mathbb{R}^{2} \mid x>0, y>0, x+y<1\right\}$ and functions $m, l, n, k:(0,1) \rightarrow \mathbb{R}$ by $m:=\alpha, l:=\beta, n:=\phi_{\mid(0,1)}$, and $k:=\phi_{\mid(0,1)}$. The functional equation $k(x+y)=m(y) n(x)+l(y)$ holds for all $(x, y) \in R$. It follows from the corollary in Aczél (2005) that either $k(x)=C x+B+P \omega$ or $k(x)=\omega \delta e^{C x}+B$ for some arbitrary parameters $B, P, C, \omega$, and $\delta$ with $C \omega \delta \neq 0$. It follows that $\phi$ is an exponential function up to positive affine transformations; by concavity, $\phi=\phi_{\theta}$ in the interior of $\mathcal{U}$. By continuity of $\phi$, this extends to the whole set $\mathcal{U}$. 


\section{A.1.5. Conclusion of the Proof}

Combining the results of Sections A.1.3 and A.1.4, $f \succsim g$ iff $\int_{S}\left(\phi_{\theta} \circ u \circ\right.$ $f) d q \geq \int_{S}\left(\phi_{\theta} \circ u \circ g\right) d q$. Because $q \in \Delta^{\sigma}$, by the variational formula (8), it follows that $f \succsim g$ iff $\min _{p \in \Delta S} \int_{S}(u \circ f) d p+\theta R(p \| q) \geq \min _{p \in \Delta S} \int_{S}(u \circ g) d p+$ $\theta R(p \| q)$.

Q.E.D.

\section{A.2. Proof of Theorem 2}

To relax the assumption of existence of certainty equivalents, the following definition will be used.

DEFINITION 5: Act $f \in \mathcal{F}_{a}(Z)$ is symmetric with respect to $E \in \Sigma_{a}$ if for all $z \in Z$,

$$
f_{E} z \sim z_{E} f
$$

Symmetric acts have the same expected utility on each "half" of the state space. ${ }^{17}$

AXIOM A5" - Second-Order Risk Aversion: If acts $f, g \in \mathcal{F}_{a}$ are symmetric with respect to $E \in \Sigma_{a}$, then for all $F \in \Sigma_{b}$,

$$
f_{F} g \sim g_{F} f \Rightarrow f_{E} g \succsim f_{F} g .
$$

The proof of Theorem 2 follows from the proof of the following stronger theorem.

THEOREM 5: Suppose $\succsim$ satisfies Axioms $\mathrm{P} 1^{\prime}-\mathrm{P} 6{ }^{\prime}$. Then Axiom A5" is satisfied if and only if the function $\phi$ in (13) is concave.

PROOF: By Theorem 3 of Ergin and Gul (2009), Axioms P1'-P6' are equivalent to $\succsim$ being represented by (13).

Q.E.D.

\section{A.2.1. Necessity}

Suppose $f \in \mathcal{F}_{a}(Z)$ is symmetric with respect to $E \in \Sigma_{a}$. Let $\alpha=q_{a}(E)$. Axiom $\mathrm{P}^{\prime}$ and representation (13) imply that there exist $z^{\prime}, z^{\prime \prime} \in Z$ with $z^{\prime} \succ z^{\prime \prime}$.

\footnotetext{
${ }^{17}$ Symmetric acts are acts that can be "subjectively mixed," that is, mixed using states rather than probabilities. Such subjective mixtures are different from subjective mixtures studied by Ghirardato, Maccheroni, Marinacci, and Siniscalchi (2003), whose construction relies on rangeconvexity of $u$. In the present setting, subjective mixtures are not needed under range-convexity of $u$.
} 
Thus, $f_{E} z^{\prime} \sim z_{E}^{\prime} f$ and $f_{E} z^{\prime \prime} \sim z_{E}^{\prime \prime} f$ imply that

$$
\begin{aligned}
& \int_{E}(u \circ f) d q_{a}+(1-\alpha) u\left(z^{\prime}\right)=\alpha u\left(z^{\prime}\right)+\int_{E^{c}}(u \circ f) d q_{a}, \\
& \int_{E}(u \circ f) d q_{a}+(1-\alpha) u\left(z^{\prime \prime}\right)=\alpha u\left(z^{\prime \prime}\right)+\int_{E^{c}}(u \circ f) d q_{a} .
\end{aligned}
$$

By subtracting (15) from (14),

$$
(1-\alpha)\left[u\left(z^{\prime}\right)-u\left(z^{\prime \prime}\right)\right]=\alpha\left[u\left(z^{\prime}\right)-u\left(z^{\prime \prime}\right)\right]
$$

thus, $\alpha=\frac{1}{2}$ and therefore

$$
\int_{E}(u \circ f) d q_{a}=\int_{E^{c}}(u \circ f) d q_{a} .
$$

Let $f, g \in \mathcal{F}_{a}(Z)$. Denote $U(f)=\int_{S_{a}}(u \circ f) d q_{a}$ and $U(g)=\int_{S_{a}}(u \circ g) d q_{a}$. If $f$ and $g$ are symmetric with respect to $E \in \Sigma_{a}$, then

$$
\begin{aligned}
\int_{E}(u \circ f) d q_{a} & =\int_{E^{c}}(u \circ f) d q_{a}=\frac{1}{2} U(f), \\
\int_{E}(u \circ g) d q_{a} & =\int_{E^{c}}(u \circ g) d q_{a}=\frac{1}{2} U(g) .
\end{aligned}
$$

Let $F \in \Sigma_{b}$ and $\beta:=q_{b}(F)$. If $f_{F} g \sim g_{F} f$, then

$$
\beta \phi(U(f))+(1-\beta) \phi(U(g))=\beta \phi(U(g))+(1-\beta) \phi(U(f)) .
$$

Thus,

$$
(2 \beta-1) \phi(U(f))=(2 \beta-1) \phi(U(g)) .
$$

If $\beta \neq \frac{1}{2}$, then $U(f)=U(g)$ and trivially

$$
\begin{aligned}
V\left(f_{E} g\right) & =\beta \phi\left(\frac{1}{2} U(f)+\frac{1}{2} U(g)\right)+(1-\beta) \phi\left(\frac{1}{2} U(f)+\frac{1}{2} U(g)\right) \\
& =\beta \phi(U(f))+(1-\beta) \phi(U(g))=V\left(f_{F} g\right) .
\end{aligned}
$$

If $\beta=\frac{1}{2}$, then

$$
\begin{aligned}
V\left(f_{E} g\right) & =\phi\left(\frac{1}{2} U(f)+\frac{1}{2} U(g)\right) \\
& \geq \frac{1}{2} \phi(U(f))+\frac{1}{2} \phi(U(g))=V\left(f_{F} g\right),
\end{aligned}
$$

where the inequality follows from concavity of $\phi$. 


\section{A.2.2. Sufficiency}

A.2.2.1. Convexity of $D_{\phi}$. Suppose $k, l \in D_{\phi}$ and $\alpha \in(0,1)$; wlog $k<l$. Let $f, g \in \mathcal{F}_{a}$ be such that $k=U(f)$ and $l=U(g)$. Define $A=\min _{s \in S} u(f(s))$ and $B=\max _{s \in S} u(g(s))$, and let $x, y \in Z$ be such that $u(x)=A$ and $u(y)=B$. By convex-rangedness of $q_{a}$, there exists $E \in \Sigma_{a}$ with $q_{a}(E)=(B-[\alpha k+$ $(1-\alpha) l])(B-A)^{-1}$. Verify that $U\left(x_{E} y\right)=\alpha k+(1-\alpha) l$. Hence, $D_{\phi}$ is a convex set.

A.2.2.2. Convexity of $\phi$. Suppose $k, l \in D_{\phi}$ and let $f, g \in \mathcal{F}_{a}$ be such that $k=U(f)$ and $l=U(g)$. Define $\underline{k}=\min _{s \in S} u(f(s)), \bar{k}=\max _{s \in S} u(f(s))$, $\underline{l}=\min _{s \in S} u(g(s))$, and $\bar{l}=\max _{s \in S} u(g(s))$. Let $\underline{x}, \bar{x}, \underline{y}, \bar{y}$ be such that $u(\underline{x})=$ $\underline{k}, u(\bar{x})=\bar{k}, u(\underline{y})=\underline{l}$, and $u(\bar{y})=\bar{l}$. Also, define $\kappa:=(\bar{k}-k)(\bar{k}-\underline{k})^{-1}$ and $\lambda:=(\bar{l}-l)(\bar{l}-\underline{l})^{-1}$. By convex-rangedness of $q_{a}$, there exist partitions $\left\{E_{1}^{\kappa}, E_{2}^{\kappa}, E_{3}^{\kappa}, E_{4}^{\kappa}\right\}$ and $\left\{E_{1}^{\lambda}, E_{2}^{\lambda}, E_{3}^{\lambda}, E_{4}^{\lambda}\right\}$ of $S_{a}$ such that $E_{1}^{\kappa} \cup E_{2}^{\kappa}=E_{1}^{\lambda} \cup E_{2}^{\lambda}$, $q_{a}\left(E_{1}^{\kappa} \cup E_{2}^{\kappa}\right)=q_{a}\left(E_{1}^{\lambda} \cup E_{2}^{\lambda}\right)=\frac{1}{2}, q_{a}\left(E_{1}^{\kappa}\right)=q_{a}\left(E_{3}^{\kappa}\right)=\frac{\kappa}{2}$, and $q_{a}\left(E_{1}^{\lambda}\right)=q_{a}\left(E_{3}^{\lambda}\right)=$ $\frac{\lambda}{2}$.

Define acts $f^{\prime}:=\underline{x} E_{1}^{\kappa} \bar{x} E_{2}^{\kappa} \underline{x} E_{3}^{\kappa} \bar{x} E_{4}^{\kappa}$ and $g^{\prime}:=\underline{y} E_{1}^{\lambda} \bar{y} E_{2}^{\lambda} \underline{y} E_{3}^{\lambda} \bar{y} E_{4}^{\lambda}$. Verify that $f^{\prime}$ and $g^{\prime}$ are symmetric with respect to $E=E_{1}^{\kappa} \cup E_{2}^{\kappa}=E_{1}^{\lambda} \cup E_{2}^{\lambda}$, and satisfy $U\left(f^{\prime}\right)=k$ and $U\left(g^{\prime}\right)=l$. By convex-rangedness of $q_{b}$, there exists $F \in \Sigma_{b}$ with $q_{b}(F)=\frac{1}{2}$. Verify that $V\left(f_{F}^{\prime} g^{\prime}\right)=\frac{1}{2} \phi(k)+\frac{1}{2} \phi(l)=V\left(g_{F}^{\prime} f^{\prime}\right)$. Hence, by Axiom $\mathrm{A} 5^{\prime}$,

$$
\phi\left(\frac{1}{2} k+\frac{1}{2} l\right)=V\left(f_{E}^{\prime} g^{\prime}\right) \geq V\left(f_{F}^{\prime} g^{\prime}\right)=\frac{1}{2} \phi(k)+\frac{1}{2} \phi(l) .
$$

As a consequence,

$$
\phi\left(\frac{1}{2} k+\frac{1}{2} l\right) \geq \frac{1}{2} \phi(k)+\frac{1}{2} \phi(l)
$$

for all $k, l \in D_{\phi}$. By Theorem 3 of Ergin and Gul (2009), the function $\phi$ is continuous on $\mathcal{D}_{\phi}$; hence, by Theorem 86 of Hardy, Littlewood, and Pólya (1952), $\phi$ is concave.

Q.E.D.

\section{A.3. Proof of Theorem 3}

By Theorem 3 of Ergin and Gul (2009), Axioms P1'-P6' are equivalent to $\succsim$ being represented by (13). Let $E \in \Sigma_{a}$ be such that $q_{a}(E)=\frac{1}{2}$. For any $v \in \mathcal{D}_{\phi}$ define a preference $\succsim^{v}$ on $\mathcal{F}$ as follows. Let $h \in \mathcal{F}_{a}$ be such that $\int_{E^{c}} u\left(h\left(s_{a}, s_{b}\right)\right) d q_{a}\left(s_{a}\right)=\frac{1}{2} v$ and for any $f, g \in \mathcal{F}(Z)$, define $f \succsim^{v} g$ iff $f_{E} h \succsim g_{E} h$. (Because of Axiom A2', the choice of particular $h$ does not mat- 
ter.) Define $\phi^{v}(u):=\phi\left(\frac{1}{2} u+\frac{1}{2} v\right)$. From representation (13), it follows that $\succsim^{v}$ is represented by

$$
\int_{S_{b}} \phi^{v}\left(2 \int_{E} u\left(f\left(s_{a}, s_{b}\right)\right) d q_{a}\left(s_{a}\right)\right) d q_{b}\left(s_{b}\right) .
$$

By Axiom A $2^{\prime}, \succsim^{v}=\succsim^{0}$ for all $v \in \mathcal{D}_{\phi}$. Hence, $\phi^{v}$ and $\phi^{0}$ are equal up to positive affine transformations, that is, $\phi\left(\frac{1}{2} u+\frac{1}{2} v\right)=\alpha(v) \phi\left(\frac{1}{2} u\right)+\beta(v)$ for all $u, v \in$ $D_{\phi}$. If $D_{\phi}$ is unbounded, then by Corollary 1 in Aczél (1966, Section 3.1.3), the function $\phi$ belongs to the exponential class.

If $D_{\phi}$ is bounded, then wlog assume that int $D_{\phi}=(0,1)$. Define the set $R:=(0,1)^{2}$. Define the functions $k:(0,2) \rightarrow \mathbb{R}$ by $k(u)=\phi\left(\frac{1}{2} u\right)$ and functions $l, m, n:(0,1) \rightarrow \mathbb{R}$ by $l:=\beta, m:=\alpha$, and $n(u)=\phi\left(\frac{1}{2} u\right)$. The functional equation $k(u+v)=m(v) n(u)+l(v)$ holds for all $(u, v) \in R$. It follows from the corollary in Aczél (2005) that either $k(u)=C u+B+P \omega$ or $k(u)=\omega \delta e^{C u}+B$ for some arbitrary parameters $a, B, P, C, \omega$, and $\delta$ with $C \omega \delta \neq 0$. Because $n$ and $k$ coincide on $(0,1)$, it follows that $\phi$ is an exponential function up to positive affine transformations in the interior of $\mathcal{U}$. By continuity of $\phi$, this extends to the whole set $\mathcal{U}$.

In both cases, $\phi$ belongs to the exponential class, that is, it is either linear, strictly concave, or strictly convex. To eliminate the last possibility, observe that Axiom A5' applied to acts $f=x_{F} y$ and $g=y_{F} x$, and events $E$ and $F$ with $q_{b}(F)=\frac{1}{2}$ and $q_{a}(E)=\frac{1}{2}$ implies that $\phi\left(\frac{1}{2} u(x)+\frac{1}{2} u(y)\right) \geq \frac{1}{2} \phi(u(x))+$ $\frac{1}{2} \phi(u(y))$ for all $x, y \in \mathcal{U}$.

It follows from Theorem 1 of Villegas (1964, Section 1) that Axiom A8' delivers countable additivity of $q_{b}$. An application of the variational formula (8) concludes the proof.

Q.E.D.

\section{REFERENCES}

Abdellaoui, M., A. Baillon, L. Placido, And P. P. Wakker (2010): "The Rich Domain of Uncertainty: Source Functions and Their Experimental Implementation," American Economic Review (forthcoming). [52]

ACZÉL, J. (1966): Lectures on Functional Equations and Their Applications. New York: Academic Press. [67,71]

(2005): "Extension of a Generalized Pexider Equation," Proceedings of the American Mathematical Society, 133, 3227-3234. [67,71]

Anderson, E. W., L. P. HAnsen, And T. J. SARGENT (2003): "A Quartet of Semigroups for Model Specification, Robustness, Prices of Risk, and Model Detection," Journal of the European Economic Association, 1, 68-123. [60]

Anscombe, F., AND R. Aumann (1963): “A Definition of Subjective Probability,” The Annals of Mathematical Statistics, 34, 199-205. [50]

BARILlas, F., L. P. HANSEN, AND T. J. SARGENT (2009): “Doubts or Variability?” Journal of Economic Theory, 144 (6), 2388-2418. [49]

Benigno, P., AND S. Nisticò (2009): “International Portfolio Allocation Under Model Uncertainty," Working Paper 14734, NBER. [49] 
CHEw, S. H., AND J. S. SAGI (2008): "Small Worlds: Modeling Attitudes Towards Sources of Uncertainty," Journal of Economic Theory, 139, 1-24. [60]

Chipman, J. (1960): "Stochastic Choice and Subjective Probability," in Decisions, Values and Groups: Proceedings. New York: Pergamon Press, 70-95. [52]

CURLEY, S., AND J. YATES (1989): "An Empirical Evaluation of Descriptive Models of Ambiguity Reactions in Choice Situations," Journal of Mathematical Psychology, 33, 397-427. [52]

DupuIS, P., AND R. S. ElLIS (1997): A Weak Convergence Approach to the Theory of Large Deviations. New York: Wiley. [56]

EINHORN, H. J., AND R. M. HOGARTH (1985): "Ambiguity and Uncertainty in Probabilistic Inference," Psychological Review, 92, 433-461. [52]

Ellsberg, D. (1961): "Risk, Ambiguity, and the Savage Axioms," The Quarterly Journal of Economics, 75, 643-669. [49,52]

ERGIN, H., AND F. GUL (2009): "A Theory of Subjective Compound Lotteries," Journal of Economic Theory, 144, 899-929. [49,50,53,58,60-63,68,70]

FISHBURN, P. C. (1970): Utility Theory for Decision Making. New York: Wiley. [50]

FOX, C., AND A. TVERSKY (1995): “Ambiguity Aversion and Comparative Ignorance," The Quarterly Journal of Economics, 110, 585-603. [52]

GhirARDATO, P., AND M. MARINACCI (2002): "Ambiguity Made Precise: A Comparative Foundation," Journal of Economic Theory, 102, 251-289. [67]

GHIRARDATO, P., F. MACCHERONI, M. MARINACCI, AND M. SinISCALCHI (2003): "A Subjective Spin on Roulette Wheels," Econometrica, 71, 1897-1908. [68]

GILBOA, I., AND D. SCHMEIDLER (1989): "Maxmin Expected Utility With Non-Unique Prior," Journal of Mathematical Economics, 18, 141-153. [48,49,53,55]

GRANT, S., AND B. POLAK (2006): "Bayesian Beliefs With Stochastic Monotonicity: An Extension of Machina and Schmeidler," Journal of Economic Theory, 130, 264-282. [56]

Grant, S., B. Polak, AND T. StrZalecki (2009): "Second-Order Expected Utility," Mimeo, Harvard University. [49,53,63,67]

Gul, F., AND W. PESENDORfER (2010): "Expected Uncertain Utility and Multiple Sources," Mimeo, Princeton University. [53,60]

HANSEN, L. P., T. SARGENT, AND T. TALLARINI (1999): "Robust Permanent Income and Pricing," Review of Economic Studies, 66, 873-907. [49]

HANSEN, L. P., T. SARGENT, AND N. WANG (2002): "Robust Permanent Income and Pricing With Filtering," Macroeconomic Dynamics, 6, 40-84. [49]

HANSEN, L. P., AND T. J. SARgENT (2001): "Robust Control and Model Uncertainty," American Economic Review, 91, 60-66. [47,53]

(2007): Robustness. Princeton: Princeton University Press. [60]

HANSEN, L. P., T. J. SARgent, G. TuRmuhambetova, AND N. Williams (2006): "Robust Control and Model Misspecification," Journal of Economic Theory, 128, 45-90. [53]

HARDY, G., J. LiTTLEWOOD, AND G. PÓlYA (1952): Inequalities. Cambridge: Cambridge University Press. [70]

HeAth, C., AND A. TVERSKY (1991): "Preference and Belief: Ambiguity and Competence in Choice Under Uncertainty," Journal of Risk and Uncertainty, 4, 5-28. [52]

JACOBSON, D. J. (1973): "Optimal Linear Systems With Exponential Performance Criteria and Their Relation to Differential Games," IEEE Transactions on Automatic Control, 18, 124-131. [57]

KARANTOunias, A. G., L. P. HANSEN, AND T. J. SARgent (2009): "Managing Expectations and Fiscal Policy," Mimeo, New York University. [49]

KLESHCHELSKI, I., AND N. VINCENT (2009): "Robust Equilibrium Yield Curves,” Mimeo, University of Montreal. [49]

KLIBANOFF, P., M. MARINACCI, AND S. MuKeRJI (2005): "A Smooth Model of Decision Making Under Ambiguity," Econometrica, 73, 1849-1892. [53]

Li, M., AND A. Tornell (2008): "Exchange Rates Under Robustness: An Account of the Forward Premium Puzzle," Mimeo, UCLA. [49] 
MACCHERONI, F., M. MARINACCI, AND A. RUSTICHINI (2004): "Ambiguity Aversion, Malevolent Nature, and the Variational Representation of Preferences," Mimeo, Collegio Carlo Alberto. [66]

(2006a): "Ambiguity Aversion, Robustness, and the Variational Representation of Preferences," Econometrica, 74, 1447-1498. [47,48,53,54]

[57]

MACHINA, M., AND D. SCHMEIDLER (1995): "Bayes Without Bernoulli: Simple Conditions for Probabilistically Sophisticated Choice," Journal of Economic Theory, 67, 108-128. [56]

Maenhout, P. (2004): "Robust Portfolio Rules and Asset Pricing," Review of Financial Studies, 17, 951-983. [49]

MARKowitz, H. M. (1952): "Portfolio Selection,” Journal of Finance, 7, 77-91. [48]

NAU, R. (2001): "Uncertainty Aversion With Second-Order Utilities and Probabilities," in Proceedings of the Second International Symposium on Imprecise Probabilities and Their Applications, available at http://www.sipta.org/isipta01/proceedings/063.html. [49,53,60]

(2006): "Uncertainty Aversion With Second-Order Utilities and Probabilities," Management Science, 52, 136-145. [49,53,60]

NeILSON, W. S. (1993): "Ambiguity Aversion: An Axiomatic Approach Using Second Order Probabilities," Mimeo. [49,53,57]

(2010): "A Simplified Axiomatic Approach to Ambiguity Aversion," Journal of Risk and Uncertainty, 41, 113-124. [49,53]

SAVAGE, L. (1972): The Foundations of Statistics. Dover. [57]

SCHMEIDLER, D. (1989): "Subjective Probability and Expected Utility Without Additivity," Econometrica, 57, 571-587. [53,62]

SKIADAS, C. (2003): "Robust Control and Recursive Utility," Finance and Stochastics, 7, 475-489. [57]

ToBIn, J. (1958): “Liquidity Preference as Behavior Toward Risk," Review of Economic Studies, 25, 65-86. [48]

VILlEGAS, C. (1964): “On Qualitative Probability $\sigma$-Algebras,” The Annals of Mathematical Statistics, 35, 1787-1796. [67,71]

WANG, T. (2003): "A Class of Multi-Prior Preferences," Mimeo, UBC. [56]

Whittle, P. (1981): "Risk-Sensitive Linear/Quadratic/Gaussian Control," Advances in Applied Probability, 13, 764-777. [57]

WoOdFord, M. (2006): "An Example of Robustly Optimal Monetary Policy With Near-Rational Expectations," Journal of the European Economic Association, 4, 386-395. [49]

Dept. of Economics, Harvard University, Littauer Center, 1805 Cambridge Street, Cambridge, MA 02138,U.S.A.; tomasz_strzalecki@harvard.edu.

Manuscript received September, 2008; final revision received August, 2010. 